\title{
Expectativa da ação da liraglutida no processo de emagrecimento
}

\author{
Expectation of liraglutide action in the weight loss process
}

\author{
Thales Henrique Martins Araújo ${ }^{1^{*}} \bullet$, Ciro José de Sousa Carvalho ${ }^{2}$
}

\author{
${ }^{1}$ Graduando do Curso de Farmácia, Faculdade de Palmas - FAPAL, Palmas, Tocantins, Brasil. ${ }^{2}$ Docente/Orientador no Curso de \\ Farmácia, Faculdade de Palmas - FAPAL, Palmas, Tocantins, Brasil. Autor para correspondência. E-mail: thalleshma@gmail.com
}

\begin{abstract}
Resumo: Introdução: A Liraglutida é um fármaco sintético, injetável, sua ação consiste no aumento da secreção de insulina, diminuindo a liberação do glucagon retardando o esvaziamento gástrico e provocando a redução do apetite. O objetivo da pesquisa foi descrever a atuação da liraglutida como estratégia farmacológica ao processo de emagrecimento, descrevendo o mecanismo de ação da liraglutida, identificando as reações adversas mais comuns associadas a este fármaco, além de apresentar os efeitos terapêuticos na perda de peso. Revisão: Trata-se de uma pesquisa de revisão integrativa da literatura, os portais para coleta de dados utilizados foram as bases da Scielo, BVS e PubMed, com textos em inglês e português do período de 2016 a 2021. Os estudos em inglês e português, com disponibilidade de texto completo. Os critérios de exclusão da pesquisa foram artigos que não se encaixaram no tema de estudo e testes em animais, combinações diretas com outros fármacos. Discussão: Evidenciou-se que o uso da liraglutida é eficiente e recomendado para o processo de emagrecimento, a dosagem mais utilizada foi de 0,6 a 3,0mg. Não havendo diferenças quanto às reações diversas em crianças, adolescentes e adultos. Considerações finais: Portanto, a liraglutida mostrou-se eficaz para a perda de peso, com alta tolerabilidade e segurança para as faixas etárias a partir de 7 anos de idade.
\end{abstract}

Palavras-chave: emagrecimento, fármacos hipoglicemiantes, liraglutida.

\begin{abstract}
Introduction: Liraglutide is a synthetic, injectable drug, its action consists in increasing insulin secretion, decreasing the release of glucagon, delaying gastric emptying and causing a reduction in appetite. The objective of the research was to describe the role of liraglutide as a pharmacological strategy for the weight loss process, describing the mechanism of action of liraglutide, identifying the most common adverse reactions associated with this drug, in addition to presenting the therapeutic effects on weight loss. Review: This is an integrative literature review research, the portals for data collection used were the bases of Scielo, BVS and PubMed, with texts in English and Portuguese from the period 2016 to 2021. Studies in English and Portuguese, with full-text availability. The exclusion criteria for the research were articles that did not fit the theme of study and tests on animals, direct combinations with other drugs. Discussion: It was evidenced that the use of liraglutide is efficient and recommended for the weight loss process, the most used dosage was from 0.6 to $3.0 \mathrm{mg}$. There are no differences regarding the different reactions in children, adolescents and adults. Final considerations: Therefore, liraglutide was shown to be effective for weight loss, with high tolerability and safety for age groups from 7 years of age.
\end{abstract}

Keywords: slimming, hypoglycemic drugs, liraglutide.

\section{Introdução}

A Organização Mundial da Saúde (OMS), destaca que a obesidade e excesso de peso como um fator preocupante pois não afeta somente os adultos, inclui-se as crianças e idosos. Em 2019, 38,2 milhões de crianças menores de 5 anos estavam sobrepeso. Em 2016 eram 1,9 bilhões de adultos sobrepeso, incluindo cerca de 650 milhões de idosos. E, cerca de $13 \%$ da população adulta do mundo eram obesos (WHO, 2021).

A obesidade e sobrepeso é caracterizado pelo armazenamento anormal de gordura, fator que pode prejudicar vários sistemas do corpo, assim prejudicando a saúde. Dado que a causa principal é a diferença entre as calorias consumidas e as calorias gastas. Que pode ser pelo aumento da ingestão de alimentos muitos calóricos especialmente ricos em açúcar e gordura. No entanto, a inatividade também contribui para o armazenamento de energia (WHO, 2021).

Neste contexto, muitos problemas de saúde podem se desencadear devido ao sobrepeso e obesidade, entre eles está a resistência periférica à insulina, fator que condiz com a hiperinsulinemia compensatória. Defende- 
se que em grande maioria dos casos, a mudança do estilo de vida com dietas e atividade física auxilia na perda de peso de forma saudável melhorando todos os sistemas do corpo. Além disso, ressalta-se que devido as complicações na saúde e a necessidade de emagrecimento, há o aumento de consumo de fármacos nesta população (Arruda et al., 2019).

Ademais, o destaque é para o Diabetes mellitus (DM), patologia classificada como crônica, e progressiva, com várias origens, apresentando hiperglicemia permanente, caracterizada pela deficiência na produção de hormônio insulina no pâncreas, ainda irregularidade na produção da resposta celular a insulina, ou melhor, a dificuldade de secreção ou da ação. A insulina tem por função manter a homeostase de glicose no corpo, assim, a produção varia da quantidade de glicose na corrente sanguínea. Outras atribuições são estímulo a lipogênese (síntese de ácidos graxos e triglicerídeos) e inibição de degradação de proteínas (Neres et al., 2019).

A DM pode se manifestar de 2 tipos principais, a tipo 1 e tipo 2 , o tipo 1 está presente em média $10 \%$ dos casos, enquanto o tipo 2 é aproximadamente $90 \%$ dos casos. Consecutivamente, a primeira é o resultado da destruição das células betas do pâncreas, ocorrendo a ausência de insulina, ocorrendo mais em crianças e jovens. Na segunda, o organismo é afetado por não realizar a transformação do açúcar em energia, isso se dá pela redução da sensibilidade dos tecidos a insulina, ou seja, a glicose não entra nas células, gerando uma serie de compensações e complicações macro e microvasculares como hipertensão, doença arterial coronariana e periférica e até acidente vascular cerebral (AVC), outras de origem renal (nefropatia) e neurológicos (perda de sensibilidade) (Ramos \& Arjona, 2013).

Atualmente, a busca pelo emagrecimento além de ser por fatores estéticos, é também porque fatores relacionados a saúde física e emocional. Há várias estratégias para um emagrecimento saudável, envolvendo muitos profissionais desde o médico, nutricionista, educador físico e farmacêutico. A busca influenciou no aumento na produção e uso de medicamentos que irão auxiliar na perda de peso corporal. Fator que, na maioria das vezes as pessoas não procuram saber e entender dos efeitos terapêuticos em sua abrangência 'somente se emagrece', e menos, interessam-se pelos efeitos adversos. A ausência de conhecimento sobre a dosagem e ação do fármaco estimula a crença que o aumento da dose irá proporcionar mais efeitos terapêuticos, prejudicando os sistemas de forma mais agressiva (Silva, Silva, \& Oyama, 2013).

Há no mercado diversas drogas para perda e peso, e a em destaque é a Liraglutida, um medicamento injetável que provoca a redução do apetite, podendo reduzir em até $10 \%$ do peso corporal, no entanto associada a atividade física e dieta saudável. A princípio, este fármaco foi legalizado para o tratamento da DM tipo 2, no entanto, por sua característica de saciedade, auxilia também no processo de emagrecimento. Dessa forma, pessoas que não possuem DM utilizam sem levar em consideração seus efeitos (Brito et al., 2013; Souza, 2012).

A Liraglutida é um fármaco sintético usado no tratamento da DM tipo 2, análogo sintético do peptídeo-1 tipo glucagon (GLP-1), que desempenha diversas funções para regular a glicose simulando o GLP-1 (secretado pelo intestino) dentro da célula. O GPL-1 composto por 30 aminoácidos, esse peptídeo é secretado juntamente com os peptídeos insulinotrópico dependente de glicose (GIP) chamados de incretinas, hormônios secretado pelo intestino delgado, que estimulam a liberação de insulina e a proliferação de células beta, o GLP atua no sistema nervoso central responsável pela regulação do apetite atrasando o esvaziamento gástrico, distendendo o estômago e assim provoca a saciedade (Pereira, 2010; Barreto, 2013).

O uso da liraglutida realiza a diminuição da glicemia em jejum e eleva a primeira fase da secreção da insulina após a alimentação, e oculta a glicemia pós-prandial. Agindo de duas formas de saciedade, através do aumento da pós-prandial de leptina e reduz o esvaziamento do estomago. É realizado uma vez por dia, pela via subcutânea, resultando na liberação de insulina de forma dependente da glicose, parando a secreção de glucagon e o esvaziamento gástrico. A concentração varia no máximo de 10 a 14 horas, isso porque sua absorção sofre atrasos devido suas características sintéticas (Morante \& Galende, 2016).

O efeito de saciedade causados pela liraglutida é iniciado por hormônio precursor da fome, a grelina, sintetizada no estomago e intestino, estimulando ao celebro (hipotálamo) o sinal da fome. Após ingerir alimentos, a grelina reduz provocando o senso de saciedade. Outro hormônio que faz parte desse processo é o colescistocinina, também produzido no intestino, mas tem ação na corrente sanguínea quando alimentos saem do estomago para o intestino. Neste caso, a leptina irar controlar a quantidade desses alimentos. Especialmente a quantidade de gordura e interrupção da ingestão, e através do sangue chega ao cérebro informando que há um estoque energético (Morante \& Galende, 2016; Júnior et al., 2012).

Estudos destacam que a com dose de 3,0 mg de liraglutida tiveram cerca de $70 \%$ de perda de peso, no entanto houve redução de hemoglobina glicada, glicemia em jejum e pressão arterial. Além de diminuir o risco de pré-diabéticos desenvolverem a DM tipo 2 (Neres et al., 2019). Portanto, a Agência Nacional de Vigilância Sanitária (ANVISA), aprovou o uso da liraglutida como um fármaco com princípio ativo como auxilio para o controle crônico de peso, juntamente com a recomendação que se tenha uma dieta saudável e a execução de 
exercícios físicos. A recomendação é para adultos com o índice de massa corporal (IMC) de 30kg/m2 para obesos e $27 \mathrm{~kg} / \mathrm{m} 2$ para os sobrepesos, além dos indivíduos que possuem DM tipo 1 e 2 (ANVISA, 2017).

Mediante o aumento do consumo desta droga, é importante destacar sobre o conhecimento do mecanismo de ação da liraglutida, sendo assim, o que leva ao questionamento: será que as pessoas que usam fármacos hipoglicemiantes como a Liraglutida para perda de peso têm conhecimento sobre seus efeitos e são suficientemente seguros quando suas expectativas de consumo? Visto pela crescente, há possibilidade de os usuários de Liraglutida desconhecerem os efeitos desse fármaco, por isso a sua realidade pode não condizer com as expectativas iniciais.

O motivo da realização da pesquisa, é dada pela grande parte dos usuários não entendem e/ou não conhecem as ações de médio e longo prazo desse fármaco, quando usado somente para o emagrecimento. E, as vezes podem nem saber que seu princípio ativo é voltado ao tratamento crônico de outra patologia. É necessário também compreender a expectativa depositada neste fármaco, em relação a real ação dele no organismo, se o mesmo apresenta malefícios ou dependências. O presente trabalho torna-se relevante em razão do elevado consumo de hipoglicemiantes por pessoas leigas e/ou que não procuram acompanhamento de um profissional qualificado para auxiliá-los no processo de emagrecimento, e pela influência da mídia, a respeito do falso conceito de beleza, podendo levar a prejuízos da saúde física e psicossociais.

O objetivo da pesquisa foi descrever a atuação da liraglutida como estratégia farmacológica ao processo de emagrecimento. Descrevendo o mecanismo de ação da liraglutida, identificando as reações adversas mais comuns associadas a este fármaco, além de apresentando os efeitos terapêuticos na perda de peso

\section{Revisão}

Este estudo trata-se de uma pesquisa de revisão integrativa da literatura, de natureza descritiva e abordagem qualitativa. Os portais para coleta de dados utilizados foram as bases da Biblioteca Eletrônica Científica Online (Scielo), Biblioteca Virtual da Saúde (BVS) e PubMed, com as palavras-chave: fármacos hipoglicemiantes, liraglutida e emagrecimento, as quais foram revisadas no DeCs (Descritores em Saúde) garantido o processamento verídico da pesquisa, com o auxílio dos booleanos and e in.

Foi pautado como critério de inclusão apenas referências dos últimos seis anos (2016 a 2021), em inglês e português, com disponibilidade de texto completo. Os critérios de exclusão da pesquisa foram artigos que não se encaixaram no tema de estudo e testes em animais, combinações diretas com outros fármacos. Assim, as variáveis abordadas pelo trabalho foram os efeitos adversos e os efeitos terapêuticos da Liraglutida, bem como algumas condições de saúde associadas a obesidade (comorbidades), principalmente a diabetes mellitus. Entretanto para a construção da introdução foram utilizados os estudos de revisão encontradas durante a seleção dos artigos nos bancos de dados.

O processo de pesquisa está representado através de um fluxograma descrito da seguinte forma, primeiramente pela identificação de artigos nas bases de dados do Scielo, BVS e PubMed, mais o total das pesquisas representadas pela cor azul. Todos os estudos excluídos durante o processo de seleção foram representados pela cor verde. E a etapas de seleção pela cor cinza até os vinte e um artigos finais pesquisas selecionadas respondendo os objetivos iniciais da pesquisa.

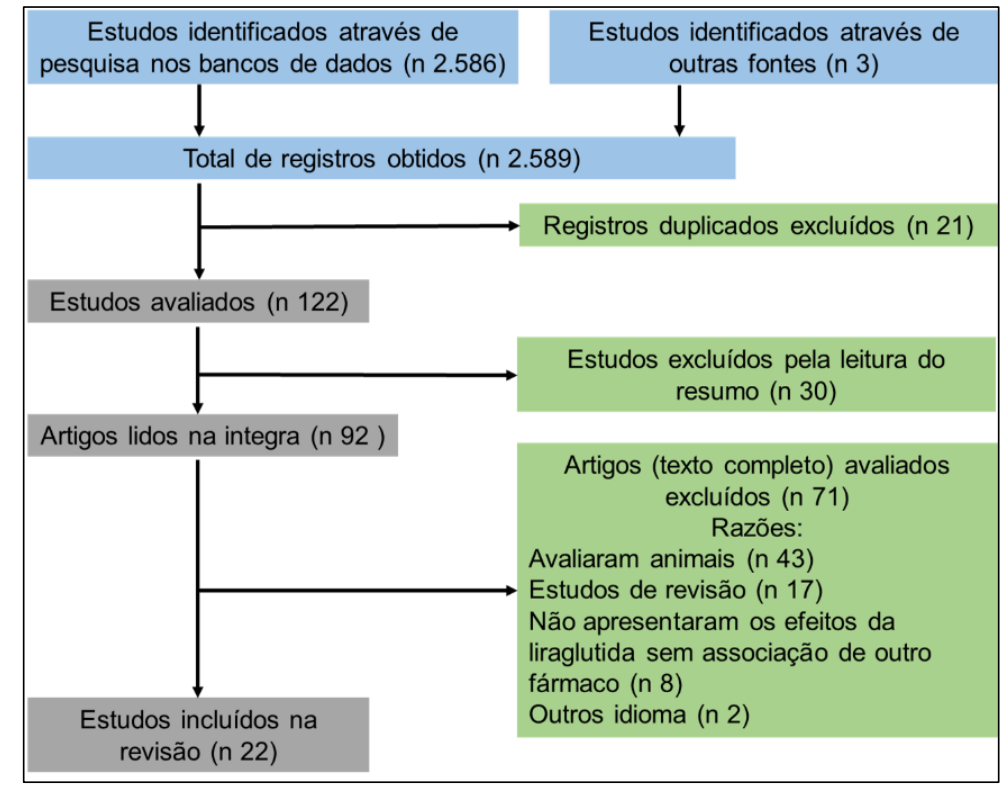

Figura 1. Fluxograma de seleção de estudos. 
A presente revisão respeitara a Resolução do Conselho Nacional de Saúde (CNS), n.466/12 (Brasil, 2012), observando se todas as literaturas utilizadas na pesquisa seguiram a resolução, cuja função é implementar normas e diretrizes regulamentadoras de pesquisas envolvendo seres humanos.

\section{Discussão}

O levantamento bibliográfico realizado foi dado o resultado de vinte e dois estudos originais sobre os efeitos da liraglutida em humanos independentes da idade. A seguir, a Tabela 1 apresenta características dos estudos.

Tabela 1. Painel do levantamento bibliográfico utilizado na construção do trabalho.

\begin{tabular}{|c|c|c|c|c|}
\hline $\mathbf{N}^{\circ}$ & Autores/Ano & Título & Natureza & Resultado \\
\hline 1 & $\begin{array}{l}\text { Blackman et al. } \\
\text { (2016) }\end{array}$ & $\begin{array}{l}\text { Efeito do liraglutida } 3,0 \mathrm{mg} \\
\text { em indivíduos com } \\
\text { obesidade e apneia } \\
\text { obstrutiva do sono } \\
\text { moderada ou grave: a escala } \\
\text { do sono Ensaio clínico } \\
\text { randomizado de apneia. } \\
\end{array}$ & $\begin{array}{c}\text { Artigo original, de } \\
\text { abordagem quantitativa, de } \\
\text { natureza aplicada, } \\
\text { exploratória, utilizando } \\
\text { procedimentos experimental } \\
\text { (randomizada e duplo-cego). }\end{array}$ & $\begin{array}{l}\text { Liraglutide produziu maior } \\
\text { porcentagem média de perda de } \\
\text { peso em comparação com placebo e } \\
\text { provoca melhora do índice de } \\
\text { apneia do sono. }\end{array}$ \\
\hline 2 & $\begin{array}{l}\text { Fujioka et al. } \\
(2016)\end{array}$ & $\begin{array}{l}\text { Prevê perda de peso precoce } \\
\text { com liraglutida } 3,0 \text { mg Perda } \\
\text { de peso em } 1 \text { ano e } \\
\text { associada a melhoria sem } \\
\text { marcadores clínicos. }\end{array}$ & $\begin{array}{c}\text { Artigo original, de } \\
\text { abordagem quantitativa, de } \\
\text { natureza aplicada, } \\
\text { exploratória, utilizando } \\
\text { procedimentos experimental } \\
\text { (multicêntricos e duplo- } \\
\text { cego). } \\
\end{array}$ & $\begin{array}{l}\text { A maior perda de peso foi nos } \\
\text { respondentes precoces nem media } \\
62,7 \% \text { (não diabéticos) a } 37,3 \% \text { dos } \\
\text { não respondentes (com DM2) em } 56 \\
\text { semanas do uso da Liraglutida. }\end{array}$ \\
\hline 3 & $\begin{array}{l}\text { Kolotkin et al. } \\
\text { (2016) }\end{array}$ & $\begin{array}{l}\text { Melhorias na qualidade de } \\
\text { vida relacionada à saúde } \\
\text { com liraglutida } 3,0 \text { mg em } \\
\text { comparação com placebo no } \\
\text { controle de peso. }\end{array}$ & $\begin{array}{c}\text { Artigo original, de } \\
\text { abordagem quantitativa, de } \\
\text { natureza aplicada, } \\
\text { exploratória, utilizando } \\
\text { procedimentos experimental } \\
\text { (randomizado e duplo- } \\
\text { cego). } \\
\end{array}$ & $\begin{array}{l}\text { Todas as subescalas do IWQOL-Lite } \\
\text { e SF-36 foram significativamente } \\
\text { melhorados com liraglutida 3,0 mg } \\
\text { vs. placebo. }\end{array}$ \\
\hline 4 & $\begin{array}{l}\text { Overgaard et al. } \\
\quad(2016)\end{array}$ & $\begin{array}{l}\text { Liraglutida 3,0 mg para } \\
\text { controle de peso: uma } \\
\text { população - Análise } \\
\text { Farmacocinética. }\end{array}$ & $\begin{array}{c}\text { Artigo original, de } \\
\text { abordagem quantitativa, de } \\
\text { natureza aplicada, } \\
\text { exploratória, utilizando } \\
\text { procedimentos experimental } \\
\text { (duplo-cego, controlado por } \\
\text { placebo, multicêntrico). } \\
\end{array}$ & $\begin{array}{l}\text { Exposição proporcional à dose de } \\
\text { liraglutida até e incluindo 3,0 mg foi } \\
\text { confirmado. Peso corporal e sexo } \\
\text { influenciou a exposição de } \\
\text { liraglutida } 3,0 \text { mg, enquanto a } \\
\text { idade, raça, etnia e estado glicêmico } \\
\text { basal não tiveram. }\end{array}$ \\
\hline 5 & $\begin{array}{l}\text { Wilding et al. } \\
\text { (2016) }\end{array}$ & $\begin{array}{l}\text { Análises de exposição- } \\
\text { resposta de liraglutida 3,0 } \\
\text { mg para peso gestão. }\end{array}$ & $\begin{array}{c}\text { Artigo original, de } \\
\text { abordagem quantitativa, de } \\
\text { natureza aplicada, } \\
\text { exploratória, utilizando } \\
\text { procedimentos experimental } \\
\text { (ensaios clínicos } \\
\text { randomizados e controlados } \\
\text { por placebo). } \\
\end{array}$ & $\begin{array}{c}\text { Houve uma resposta clara à } \\
\text { exposição à perda de peso. A perda } \\
\text { de peso aumentou com a maior } \\
\text { exposição e pareceu estabilizar nas } \\
\text { exposições mais alta associado a } \\
\text { liraglutida 3,0 mg na maioria dos } \\
\text { indivíduos. }\end{array}$ \\
\hline 6 & $\begin{array}{c}\text { Danne et al. } \\
\text { (2017) }\end{array}$ & $\begin{array}{c}\text { Liraglutida em uma } \\
\text { população adolescente com } \\
\text { obesidade: Um ensaio } \\
\text { randomizado, duplo-cego e } \\
\text { controlado por placebo de } 5 \\
\text { semanas para avaliar a } \\
\text { segurança, tolerabilidade e } \\
\text { farmacocinética do } \\
\text { liraglutida em Adolescentes } \\
\text { com } 12 \text { a } 17 \text { anos. }\end{array}$ & $\begin{array}{c}\text { Artigo original, de } \\
\text { abordagem quantitativa, de } \\
\text { natureza aplicada, } \\
\text { exploratória, utilizando } \\
\text { procedimentos experimental } \\
\text { (ensaio randomizado, } \\
\text { duplo-cego e controlado por } \\
\text { placebo). }\end{array}$ & $\begin{array}{l}\text { Todos os participantes que } \\
\text { receberam liraglutida e } 4 \text { que } \\
\text { receberam placebo (57,1\%) tiveram } \\
\text { pelo menos } 1 \text { evento adverso. A } \\
\text { maioria eventos adversos } \\
\text { emergentes do tratamento comuns } \\
\text { foram distúrbios gastrointestinais. } \\
\text { A liraglutida teve um perfil de } \\
\text { segurança e tolerabilidade } \\
\text { semelhante em comparação com os } \\
\text { adultos quando administrado a } \\
\text { adolescentes com obesidade. }\end{array}$ \\
\hline 7 & $\begin{array}{l}\text { Le Roux et al. } \\
\text { (2018) }\end{array}$ & $\begin{array}{c}\text { Comparação de eficácia e } \\
\text { segurança de Liraglutida 3,0 } \\
\text { mg em indivíduos com IMC } \\
\text { acima e abaixo de } 35 \mathrm{~kg} / \\
\mathrm{m}^{2} \text { : Uma Análise Post-hoc. } \\
\end{array}$ & $\begin{array}{c}\text { Artigo original, de } \\
\text { abordagem quantitativa, de } \\
\text { natureza aplicada, } \\
\text { exploratória, utilizando } \\
\text { procedimentos experimental } \\
\end{array}$ & $\begin{array}{c}\text { A perda de peso foi } \\
\text { significativamente maior (0-56 } \\
\text { semanas) observada com liraglutida } \\
\text { 3,0 mg versus placebo em todos os } \\
\text { grupos de pacientes enquanto em }\end{array}$ \\
\hline
\end{tabular}




\begin{tabular}{|c|c|c|c|}
\hline & & & $\begin{array}{c}\text { (randomizados, duplo- } \\
\text { cegos, ensaios controlados } \\
\text { com placebo). }\end{array}$ \\
\hline 8 & $\begin{array}{l}\text { Iepsen et al. } \\
(2018)\end{array}$ & $\begin{array}{c}\text { Pacientes com obesidade } \\
\text { causada por melanocortina- } \\
\text { 4Mutações no receptor } \\
\text { podem ser tratadas com um } \\
\text { Agonista do receptor do } \\
\text { peptídeo-1 semelhante ao } \\
\text { glucagon. }\end{array}$ & $\begin{array}{c}\text { Artigo original, de } \\
\text { abordagem quantitativa, de } \\
\text { natureza aplicada, } \\
\text { exploratória, utilizando } \\
\text { procedimentos experimental } \\
\text { (ensaio randomizado). }\end{array}$ \\
\hline 9 & $\begin{array}{c}\text { Kochar \& Sethi } \\
\text { (2019) }\end{array}$ & $\begin{array}{l}\text { Eficácia e segurança do } \\
\text { liraglutida em adolescentes } \\
\text { indianos com obesidade. }\end{array}$ & $\begin{array}{c}\text { Artigo original, de } \\
\text { abordagem quantitativa, de } \\
\text { natureza aplicada, } \\
\text { exploratória, utilizando } \\
\text { procedimentos experimental } \\
\text { (não randomizado e aberto). }\end{array}$ \\
\hline
\end{tabular}

tratamento. Não houve evidência de

que o efeito de redução de peso do

liraglutida 3,0 mg entre os subgrupos de IMC.

liraglutida induziu um igual,

clinicamente significativa perda de

peso de $6 \%$ em ambos os grupos,

indicando que o efeito redutor do

apetite do liraglutida é pré-servido

em MC4R obesidade causal e que Liraglutide atua

independentemente da via MC4R.

Houve uma diminuição significativa

no peso corporal, IMC, jejum, $1 \mathrm{~h}$

após tolerância à glicose $\mathrm{e}$

tolerância à glicose de 2 horas. As

mudanças desde a linha de base até

o final de estudo foram para peso

corporal - 6,5 $\pm 4,2 \mathrm{~kg}$ e IMC $-2,35 \pm$ $1,30 \mathrm{~kg}$.

$\begin{array}{ccc}\text { Kolotkin et al. } & \text { Melhorias na qualidade de } & \text { Artigo original, de } \\ (2018) & \text { vida relacionada à saúde ao } & \text { abordagem quantitativa, de } \\ & \text { longo3 anos com liraglutida } & \text { natureza aplicada, } \\ & 3,0 \text { mg em comparação com } & \text { exploratória, utilizando } \\ & \text { placebo em participantes } & \text { procedimentos } \\ & \text { com sobrepeso ou } & \text { experimental. }\end{array}$

$\begin{array}{ccc}\text { Mastrandrea et } & \text { Efeitos do liraglutida em } & \text { Artigo original, de } \\ \text { al. (2019) } & \text { uma população pediátrica (7 } & \text { abordagem quantitativa, de } \\ & -11 \text { anos) com obesidade: } & \text { natureza aplicada, } \\ & \text { um estudo randomizado, } & \text { exploratória, utilizando } \\ & \text { duplo - cego, placebo - } & \text { procedimentos experimental } \\ & \text { controlado, curto -ensaio de } & \text { (ensaio randomizado, } \\ & \text { prazo para avaliar a } & \text { duplo-cego, controlado por } \\ & \text { segurança, tolerabilidade, } & \text { placebo). }\end{array}$

Liraglutida 3,0 mg, mais dieta e

exercício, está associada com

melhorias de longo prazo na

Qualidade de Vida Relacionada à

Saúde (QVRS) com obesidade ou

sobrepeso com comorbidade vs. placebo.

Uma redução significativa no índice de massa corporal (IMC) pontuação

$\mathrm{Z}$ da linha de base ao final do

tratamento (diferença estimada de tratamento: $-0,28 ; \mathrm{P}=0,0062$ ) foi observada.

segurança, tolerabilidade, farmacocinética farmacodinâmica.

\begin{tabular}{|c|c|c|c|}
\hline 12 & $\begin{array}{c}\text { Chao et al. } \\
\text { (2019) }\end{array}$ & $\begin{array}{l}\text { Efeitos da liraglutida e da } \\
\text { perda de peso } \\
\text { comportamental nos } \\
\text { alimentos Desejos, } \\
\text { comportamentos } \\
\text { alimentares e transtorno } \\
\text { alimentar Psicopatologia. }\end{array}$ & $\begin{array}{c}\text { Artigo original, de } \\
\text { abordagem quantitativa, de } \\
\text { natureza aplicada, } \\
\text { exploratória, utilizando } \\
\text { procedimentos experimental } \\
\text { (randomizado e } \\
\text { multicomponente). }\end{array}$ \\
\hline 13 & $\begin{array}{l}\text { Cuomo et al. } \\
\text { (2019) }\end{array}$ & $\begin{array}{c}\text { Viabilidade, adesão e } \\
\text { eficácia de Tratamento com } \\
\text { liraglutida em uma amostra } \\
\text { de Indivíduos com } \\
\text { Transtornos do Humor e } \\
\text { Obesidade. }\end{array}$ & $\begin{array}{c}\text { Artigo original, de } \\
\text { abordagem quantitativa, de } \\
\text { natureza aplicada, } \\
\text { descritiva, utilizando } \\
\text { procedimentos } \\
\text { observacional } \\
\text { (retrospectivo). }\end{array}$ \\
\hline
\end{tabular}

\begin{tabular}{|c|c|c|c|c|}
\hline 14 & $\begin{array}{l}\text { Papamargaritis } \\
\text { et al. (2020) }\end{array}$ & $\begin{array}{c}\text { Eficácia e custo de } \\
\text { integração de um caminho } \\
\text { pragmático para prescrever } \\
\text { liraglutida } 3,0 \mathrm{mg} \text { em } \\
\text { serviços de obesidade } \\
\text { (Estudo STRIVE): protocolo } \\
\text { de estudo de um rótulo } \\
\text { aberto, mundo real, } \\
\text { randomizado, ensaio } \\
\text { controlado. }\end{array}$ & $\begin{array}{l}\text { Artigo original, de } \\
\text { abordagem quantitativa, de } \\
\text { natureza aplicada, } \\
\text { exploratória, utilizando } \\
\text { procedimentos experimental } \\
\text { (um rótulo aberto, mundo } \\
\text { real, randomizado, ensaio } \\
\text { controlado). }\end{array}$ & $\begin{array}{l}\text { Principal: é comparar a proporção } \\
\text { de participantes que alcançaram } \\
\text { uma perda de peso } \geqslant 15 \% \text { em } 52 \\
\text { semanas com uma via de prescrição } \\
\text { direcionada versus padrão Cuidado. } \\
\text { Secundários incluem uma } \\
\text { comparação de (1) manutenção da } \\
\text { perda de peso em } 104 \text { semanas e (2) } \\
\text { o orçamento impacto e eficácia de } \\
\text { custo entre os dois grupos em uma } \\
\text { configuração do mundo real. }\end{array}$ \\
\hline 15 & $\begin{array}{l}\text { Peradze et al. } \\
\text { (2019) }\end{array}$ & $\begin{array}{l}\text { Tratamento de curto prazo } \\
\text { com alta dose liraglutida } \\
\text { melhora o perfil de lipídios e } \\
\text { lipoproteínase altera os }\end{array}$ & $\begin{array}{c}\text { Artigo original, de } \\
\text { abordagem quantitativa, de } \\
\text { natureza aplicada, } \\
\text { exploratória, utilizando }\end{array}$ & $\begin{array}{l}\text { As concentrações de marcadores } \\
\text { cardiovasculares importantes, como } \\
\text { colesterol total, livre e } \\
\text { remanescente foram reduzidos com }\end{array}$ \\
\hline
\end{tabular}




\begin{tabular}{|c|c|c|c|}
\hline & & $\begin{array}{l}\text { mediadores hormonais de } \\
\text { lipídios metabolismo em } \\
\text { pacientes obesos sem } \\
\text { manifestação diabetes } \\
\text { mellitus tipo 2: um } \\
\text { randomizado, controlado } \\
\text { por placebo, cruzado, duplo- } \\
\text { cego ensaio clínico. }\end{array}$ & $\begin{array}{l}\text { procedimentos experimental } \\
\text { (randomizado, controlado } \\
\text { por placebo, cruzado, duplo- } \\
\text { cego ensaio clínico). }\end{array}$ \\
\hline 16 & $\begin{array}{l}\text { Tronieri et al. } \\
\text { (2020) }\end{array}$ & $\begin{array}{l}\text { Efeitos da liraglutida no } \\
\text { apetite, preocupação } \\
\text { alimentar e Gostos de } \\
\text { comida: resultados de um } \\
\text { ensaio clínico randomizado } \\
\text { e controlado. }\end{array}$ & $\begin{array}{c}\text { Artigo original, de } \\
\text { abordagem quantitativa, de } \\
\text { natureza aplicada, } \\
\text { exploratória, utilizando } \\
\text { procedimentos experimental } \\
\text { (ensaio piloto randomizado } \\
\text { controlado). }\end{array}$ \\
\hline 17 & $\begin{array}{l}\text { Chou \& Chuang } \\
\text { (2020) }\end{array}$ & $\begin{array}{c}\text { Avaliação da eficácia da } \\
\text { dose baixa liraglutida no } \\
\text { controle de peso entre } \\
\text { taiwaneses pacientes sem } \\
\text { diabetes. }\end{array}$ & $\begin{array}{c}\text { Artigo original, de } \\
\text { abordagem quantitativa, de } \\
\text { natureza aplicada, } \\
\text { descritiva, utilizando } \\
\text { procedimentos } \\
\text { observacional } \\
\text { (retrospectivo). }\end{array}$ \\
\hline
\end{tabular}

Eficácia clínica no mundo

\section{real do liraglutida 3,0mg para controle de peso no \\ (2019)

$$
\begin{array}{r}
\text { para controle de } \\
\text { Canadá. }
\end{array}
$$

\begin{tabular}{cc}
\hline 18 Wharton et al. & $\begin{array}{c}\text { Eficácia clínica no mundo } \\
\text { (2019) }\end{array}$ \\
& $\begin{array}{c}\text { real do liraglutida 3,0mg } \\
\text { para controle de peso no } \\
\text { Canadá. }\end{array}$
\end{tabular}

\begin{tabular}{ccc}
\hline & Tronieri et al. & Efeitos do Liraglutide Plus \\
(2019) & Phentermine em Adultos \\
& com Obesidade Após 1 ano \\
& de tratamento apenas com \\
& liraglutida: A Ensaio Piloto \\
& Randomizado Controlado \\
& por Placebo.
\end{tabular}
liraglutida antes e depois do ajuste
para perda de peso.

Na semana 52, os participantes

tratados apenas com IBT perderam

$6,2 \pm 1,6 \%$ do peso inicial,em

comparação com $11,8 \pm 1,6 \%$ e 12,1

$\pm 1,5 \%$ nos grupos IBT-liraglutida e multicomponentes, respectivamente.

Com 12 semanas de uso da medicação, ambos os grupos apresentaram peso estatístico redução. Doses mais altas de liraglutida tiveram melhor eficácia e $44,4 \%$ dos pacientes no liraglutida $1,2 \mathrm{mg}$ grupo atingiu redução de peso evidente, enquanto apenas

$32,1 \%$ no grupo de $0,6 \mathrm{mg}$ conseguiu isso.

Artigo original, de
abordagem quantitativa, de
natureza aplicada,
descritiva, utilizando
procedimentos
observacional
(retrospectivo).

Artigo original, de abordagem quantitativa, de natureza aplicada, exploratória, utilizando procedimentos experimental (ensaio piloto randomizado controlado por placebo).
Lá foi uma mudança significativa no peso corporal 6 e 4 meses após o início do tratamento em indivíduos persistentes ( $\geqslant 6$ meses: $-8,0 \mathrm{~kg}, \mathrm{P}$ $<0,001$; $\geqslant 4$ meses: $-7,0 \mathrm{~kg}, \mathrm{P}<0,001$ ) e em todos os indivíduos, independentemente da persistência $(-7,3 \mathrm{~kg} ; \mathrm{P}<0,001)$.

Na semana 12, os grupos

liraglutida-fentermina e liraglutidaplacebo perderam uma média $( \pm$ SEM) de $1,6 \pm 0,6 \%$ e $0,1 \pm 0,5 \%$ do peso da randomização, respectivamente $(p=0,073)$. Os participantes da fentermina geralmente relataram grandes reduções na fome e na preocupação com alimentos do que os participantes com liraglutidaplacebo durante as primeiras 8 semanas do estudo de extensão. O tratamento do mundo real com liraglutida3,0 mg, independentemente da classe de obesidade, está associado a similar clinicamente significativo perda de peso, tempo de manutenção da dose e persistência da medicação. Um modelo farmacocinético / farmacodinâmico forneceu uma descrição adequada das trajetórias de perda de peso para todas as doses estudadas. Sexo e diabetes foram identificados como as covariáveis mais influentes. resultados de perda de peso modelo-abordagem baseada.

Artigo original, de abordagem quantitativa, de natureza aplicada, exploratória, utilizando procedimentos experimental (ensaios clínicos randomizados e controlados de fase II e III; duplo-cego, placebo-controlado).

\begin{tabular}{c}
\hline $22 \quad$ Park et al. \\
(2021)
\end{tabular}

Eficácia clínica do

liraglutida na perda de pesonos sul-coreanos primeiros dados retrospectivos do mundo real sobre Saxenda na Ásia

Artigo original, de

abordagem quantitativa, de natureza aplicada, descritiva, utilizando procedimentos observacional (retrospectivo).
O tratamento com liraglutida levou à perda significativa de peso em pacientes sul-coreanos, e a redução da massa gorda foi proeminente durante o tratamento. A perda de peso não foi significativamente relacionada à idade, sexo, IMC




\section{Mecanismo de ação da liraglutida}

Dentre as características dos mecanismos de ação da liraglutida está sua alta compatibilidade com o Glucagon em média 97\% em relação ao humano, representando eficácia e concernente aos fatores que envolve o processo para controle glicêmico. Podendo também ser chamado de regulador 'fisiológico' do apetite. Sobre a exposição da liraglutida, Wharton et al. (2020) e Wilding et al. (2016), descrevem a atuação do fármaco, em que GLP-1 agem como hormônios no intestino reduzindo o glucagon, bem como o processo de digestão, assim dando a sensação de se saciedade.

Visto que a liraglutida é um análogo do hormônio incretina intestinal, resposta à ingestão de alimentos, o GLP-1 é secretado pelas células L intestinais e reduz a glicose no sangue ao inibir a secreção de glucagon e promover secreção de insulina de forma dependente da glicose. Pertencente a classe dos agonistas do receptor de GLP-1 que estimulam a perda de peso por meio de reduções no apetite e na ingestão de energia (Mastrandrea et al., 2019).

A liraglutida parece agir no sistema nervoso central para diminuir o peso corporal por meio do aumento da saciedade, com redução concomitante na ingestão de calorias. Em resposta a altamente desejável aos alimentos, os agonistas do receptor de GLP diminuem a ativação em áreas do cérebro associadas com o apetite e recompensa, como o córtex parietal, ínsula, putâmen e córtex orbito frontal. Isso sugere que o liraglutida pode reduzir a recompensa alimentar, como o desejo por comida, um intenso desejo de consumir um determinado alimento. A liraglutida também pode melhorar outros aspectos do apetite e comportamentos alimentares, como fome, restrição alimentar, desinibição, compulsão alimentar e preocupações cognitivas relacionadas à alimentação, forma e peso (Chao et al., 2019).

Segundo Kolotkin et al. (2016) a dose da liraglutida é geralmente entre $1,8 \mathrm{mg}$ a 3,0mg dose utilizada quando o objetivo é perda de peso, uma vez ao dia, destacando a que sua maior característica está na redução de apetite e ingestão de energia em vez do aumento o gasto energético, além disso, auxilia na melhoria de diversas comorbidades. Nesta perspectiva, Overgaard et al. (2016) relatam que a dosagem de 1,8mg é mais utilizada para DM2 e que a dosagem de 3,0mg para perda de peso, pois esta medida mostrou envolver o aumento dos sinais de saciedade hipotalâmica e ao mesmo tempo desestimular os sinais de estimulação do apetite proporcionando menor ingestão de energia.

Outras características são apresentadas por Wilding et al. (2016) a respeito dos fatores intrínsecos e extrínsecos que afetam a depuração e assim a concentração plasmática em estados estacionários como o sexo, peso e outros, por esse motivo a diversas pesquisas e estudos sobre a dosagem ideal da liraglutida para a perda de peso em curto e longo prazo, ou ainda doses para manutenção do peso e consumo diário para diabetes.

Iepsen et al. (2018) apontam a respeito de um dos tipos de obesidade, a causada pelo receptor de melanocortina-4 (MC4R) - localizado no sistema nervoso central, receptor que tem a função de diminuir o apetite quando o indivíduo já se alimentou, no entanto, uma vez que são inibidos, aumentam a sensação de fome. Primeiramente, a discussão se deu pela possibilidade de haver diferenças quanto ao mecanismo de ação da liraglutida em referência a obesidade influenciada por estilo de vida (obesidade comum), em relação a MC4R. Mas, notou-se que não há diferenças entre os grupos com obesidade comum a grupo com obesidade pelo MC4R, evidenciando que mesmo apresentando da distinção dos estímulos iniciais da sensação de fome, não houve diferenças significativas ao mecanismo de ação da liraglutida. Dado também a afirmativa que os pacientes responderam que possivelmente conseguiram manter o peso com o uso da medicação (liraglutida), devido aos baixos índices de efeitos adversos. O autor solicita mais pesquisas que possam avaliar o uso da liraglutida em outros tipos de obesidade.

\section{Reações adversas mais comuns}

A respeito as reações adversas, vários autores relatam sobre a ocorrência delas no período de consumo dos participantes, assim, na pesquisa de Le Roux et al. (2018) os efeitos da utilização de 3,0mg de liraglutida foram semelhantes nos dois grupos com liraglutida e o placebo. Foi observado a exposição e preocupação com a taxa de distúrbios na vesícula biliar, que se apresentou de baixo risco, assim também com o risco de pancreatite aguda, também de baixo risco. O relato mais comum entre os grupos foram os gastrointestinais, sem diferenças significativas entre eles. No entanto, vale ressaltar que os índices foram mais relevantes no grupo liraglutida, que no placebo. Outros efeitos foram observados como a frequência cardíaca e as taxas de 
hipoglicemia que também tiveram a mesma relação com os anteriores, ou seja, presente de forma mais significativa no grupo de liraglutida. Efeitos similarmente foram apresentados no estudo de Iepsen et al. (2018), acrescentando apenas os no trato gastrointestinais, de aspecto leve e passageiro, em forma principalmente de náuseas envolvendo a metade dos participantes, sem mais relatos de outros efeitos.

No estudo de Danne et al. (2017) realizado com adolescentes obesos, observou-se que todos os participantes tiveram ao menos 1 efeito adverso chamados de eventos adversos emergentes do tratamento (TEAEs). O estudo foi realizado com 21 adolescentes com idade de 12 a 17 anos, com IMC maior ou igual a $30 \mathrm{~kg} / \mathrm{m} 2$. Receberam a dosagem foi inicialmente de $0,6 \mathrm{mg}$ e gradualmente sendo acrescida para até no máximo 3,0mg na última semana por 5 semanas, os principais efeitos formam os gastrointestinais e de hipoglicemia, sem nenhum episódio grave, não havendo problemas inesperados. Assim, pode ser observado que os efeitos de tolerabilidade foram semelhantes aos dos adultos, assim é assegurado que a dosagem de adultos é aprovada e tolerados por adolescentes obesos.

No estudo de Mastrandrea et al. (2019) visando o tratamento da obesidade infantil, sendo de grande preocupação devido a limitação, este autor teve por objetivo realizar a avaliação e segurança, tolerabilidade, farmacocinética e farmacodinâmica durantes o tratamento de curto prazo com a liraglutida. As crianças tinham entre 7 e 11 anos, com média de peso de $71 \mathrm{~kg}$. Foram relatados mais de 35 efeitos adversos pelo uso da liraglutida enquanto o placebo foi em torno de 12 efeitos. Os que mais se destacaram foram as do trato gástrico intestinais, cerca de $37 \%$ dos participantes com sintomas leve, 3 participantes com gravidade moderada e nenhum participante teve efeitos grave. Houve em 5 pacientes episódios de hipoglicemia assintomática que em 4 desses usavam a liraglutida. A exposição a liraglutida foi consistente e a covariável foi o peso corporal, sendo verificado a redução do IMC na fase final do tratamento, o que os autores asseguram que em curto prazo a liraglutida reduziu $-0,28$, o que ressalva que a droga é confiável e segura de boa tolerabilidade semelhantes aos ensaios realizados com adultos e adolescentes.

Papathanasiou et al. (2020), investigaram o impacto da perda de peso associados também a problemas gástricointestinais. $\mathrm{O}$ tratamento durou 52 semanas e foram analisados as covariáveis como peso corporal, hemoglobina glicada, idade, gênero, diabetes, raça e religião. Assim, o sexo e o diabetes foram as covariáveis mais influentes no estudo. A relação entre a exposição sistêmica, liraglutida e perda de peso foi estabelecido com sucesso. Ainda, o estudo previu o ganho de peso, após interrupção do uso deste fármaco. O autor sugere que os problemas gástricointestinais podem ser mitigadospor escala mais lenta, ou seja, fazer o estudo por mais tempo, com dosagens selecionadas e com mínimo impacto possível no processo de perda de peso.

Na pesquisa de Wilding et al. (2016), foi observado além das náuseas, um episódio de vômito em um estudo com durabilidade de 56 semanas que pode ter sido impulsionado pela dosagem de liraglutida de 3,0 mg. Outra situação vivenciada foram ocorrências de hipoglicemia sintomática, as quais foram documentadas nos pacientes do grupo de DM2. Neste estudo, os indivíduos com distúrbios na vesícula biliar, pancreatite aguda, neoplasias benignas não tiveram exposição a liraglutida como a população geral, pois foram avaliados minuciosamente a fim de adequarem a dosagem certa do fármaco. Por meio da metanálise, não foram notadas aumento significativo de níveis de citonina (proteína que auxilia células aos seus receptores) nos ensaios em nenhum dos grupos, ademais houve um leve aumento na frequência cardíaca dos participantes que realizaram uso da liraglutida.

Em concordância, para Fujioka et al. (2016), um dos pontos em destaque da sua pesquisa, foi as alterações cardiometabólicas, principalmente a frequência cardíaca, que aumentou cerca de 2,6 bpm, e os mesmos sintomas gastrointestinais das outras pesquisas, náuseas e enjoos.O objetivo da pesquisa foi identificar os critérios de resposta precoce para prever a perda de peso de 5\% com a liraglutida de 3,0 mg por 56 semanas. Durante todo o período de estudo a maior perda média de peso foi observada no grupo de respondentes precoce versus os não respondentes iniciais: $10,8 \%$ versus $3,0 \%$ (sem DM2) e $8,5 \%$ versus $3,1 \%$ (com DM2).

\section{Efeitos terapêuticos da liraglutida na perda de peso}

Blackman et al. (2016) destacam que na pesquisa realizada durante 32 semanas, houve a redução do peso com o uso da liraglutida e também houve a redução a apneia obstrutiva do sono (condição associada a obesidade), a diferença com o placebo foi em média de $-4,2 \%$ no que ocorreu uma considerada melhora nos desfechos da apneia. Outras características que tiveram reduções foram a hemoglobina glicada, a pressão arterial sistólica, mas destaque é para o perfil de segurança da droga em quantidades de 3,0 $\mathrm{mg}$ a qual foi semelhante a dosagem de $1,8 \mathrm{mg}$. Ao ser associada com a dieta e exercícios, a liraglutida foi bem tolerada e provocou maiores reduções no peso, pressão arterial e hemoglobina glicada, melhorando os sintomas da dispneia. 
Kolotkin et al. (2016) por meio das escalas do IWQOL-Lite e SF-36 verificaram se a liraglutida poderia proporcionar a melhora na qualidade de vida dos participantes sobrepeso. Por fim, soube-se que todas as escalas tiveram melhoras mediante a usabilidade da liraglutida 3,0 mg em relação ao placebo, no entanto, é importante acrescentar que os participantes tiveram auxilio nutricional de menos 500Kcal e acompanhamento para os exercícios físicos.

Overgaard et al. (2016) tiveram como foco de pesquisa, os desfechos como o peso, sexo, idade, raça, etnia, bem como o estado glicêmico dos participantes poderiam ou não influenciar na perda de peso usando a liraglutida. Assim, a exposição a liraglutida de 3,0 mg sofreu influência quanto ao peso corporal e ao sexo. Em quanto a idade, raça, etnia e o estado glicêmico não houveram influências. As estimativas é que mais de $40 \%$ menos efetiva em pessoas com o peso aproximado de $230 \mathrm{~kg}$ que de $100 \mathrm{~kg}$, e menos efetiva em mulheres que em homens com a mesma quantidade de peso. Não houve influências na função renal. $O$ autor sugere que mais pesquisas com critérios parecidos possam ser realizadas para melhor exposição respostas do fármaco.

No estudo de Wharton et al. (2019) com o objetivo de analisar a eficácia clínica do da liraglutida 3,0 mg, em combinação com dieta e exercícios, foram investigados 4 e 6 meses após o início. Viu-se que a redução média do peso dos participantes foram $8 \mathrm{~kg}$, no prazo de 6 meses, e os que ficaram durante 4 meses também apresentaram perda de peso significativa. Visto que o único meio de regressão logística foi a idade, atingindo menos de $5 \%$ de perda de peso. Notou-se diminuição significativa de $0,4 \%$ nos níveis de hemoglobina glicada e diminuição na pressão arterial sistólica em 3,0 mmHg.

Na pesquisa de Kolotkin et al. (2018), o efeito da liraglutida quanto ao peso corporal ao longo do tempo (160 semanas), apontou eficácia ao grupo que consumiu o fármaco, obteve resultado significante quanto ao grupo placebo. A diferença em média foi de $11 \%$, assim analisou-se os resultados quanto ao impacto no pela qualidade de vida-lite (IWQOL-Lite) verificado que no início havia muitas semelhanças entre os grupos, mas aos 3 anos de estudo, notou-se mudanças nas linhas base do IWQOL-Lite de forma benéfica para os participantes do grupo liraglutida. O mesmo aconteceu com os resultados do Short-Form 36, que no início tinham os valores semelhantes, mas foram melhorando no decorrer do tempo, mostrando que os aspectos de melhor influencia foram na escala de funcionamento físico, englobando saúde geral, vitalidade e saúde mental, e outras diferenças significativas como dores no corpo, funcionamento social e emocional.

No estudo de Kochar \& Sethi (2019) realizado com adolescentes indianos, pois as principais intervenções realizadas para a obesidade em crianças e adolescentes são muito limitadas em três modos principais de intervenção que são o estilo de vida, farmacoterapia e cirurgia bariátrica. Envolvendo a modificação desses componentes em: restrição calórica, aumento da atividade física e aconselhamento comportamental para melhor relacionamento com a comida. $\mathrm{O}$ objetivo da pesquisa foi avaliar a eficácia e segurança do liraglutida em adolescentes com obesidade. o estudo foi desenvolvido durante 12 semanas com a associação ao jejum de 1 após a tolerância da glicose e depois a jejum a $2 \mathrm{~h}$. Pôde-se constatar a diminuição da pressão sistólica e diastólica, melhora nas enzimas hepáticas e do colesterol e triglicerídeos. A pesquisa foi condizente que a liraglutida é eficiente e é uma alternativa segura para os adolescentes que não estão respondendo a outras modalidades para perda de peso.

Para Iepsen et al. (2018), no processo de 16 semanas de tratamento com o uso da liraglutida houve diminuição no peso corporal, mais especificamente, diminuição na porcentagem de gordura na cintura e quadril, tanto nos pacientes que apresentam mutações MC4R, quanto nos participantes do grupo controle, as diferenças em porcentagem respectivamente foi de $-5,7 \%$ e $-5,5 \%$, observou-se que durante esse período não houve diminuição da massa magra. Foi investigado também sobre a melhora da glicose e regulação desta, onde os resultados foram significativos, $47,5 \%$ no grupo MC4R, e $24 \%$ no grupo controle. Bem como, o resultado da concentração de lipídios (colesterol plasmático e triglicerídeos) que se mostrou significativo somente no grupo controle. $\mathrm{O}$ autor descreve alguns benefícios gerais para a saúde no tratamento como a perda de peso (massa gorda) e não a magra, redução de medidas, melhoras na insulina, diminuição de fatores de risco cardiometabólicas (principalmente a pressão arterial), ainda relatando sua eficácia a proteção à prédiabetes.

Para Chao et al. (2019), os efeitos da liraglutida devem ser verificados sendo associados com desejos e comportamentos alimentares e psicopatologia. Com isto, no período de 24 a 52 semanas são realizados ensaios com a terapia comportamental intensiva com e sem o uso da liraglutida. Além disso, foi realizado uma dieta com déficit calórico por 12 semanas com a ingestão de 1000 a $1200 \mathrm{kcal}$. Os grupos com o uso da liraglutida relataram quedas no peso em relação ao que realizaram a terapia comportamental, relatando sobre a significante desinibição alimentar, transtorno alimentar e outros aspectos da compulsão alimentar, no entanto as mudanças não foram tão significativas na semana 52. Assim, a combinação com a liraglutida e 
terapia computacional foi associada a maior em curto prazo nas melhorias da desinibição e transtornos e preocupações que somente com a terapia.

Em condições diferentes que o usual, a pesquisa de Cuomo et al., (2019), afim de verificar a viabilidade adesão e eficácia do tratamento da obesidade com a liraglutida em indivíduos com transtornos de humor, assim 29 pacientes com transtorno bipolar ou depressivo maior foram submetidos uma vez por dia por via subcutânea a receber gradualmente doses de 0,6 a 3,0 mg de liraglutida, todos obesos e com histórico de falhas com intervenções com exercícios e dietas. Os pesos dos pacientes foram registrados no início, após 1 mês, 3 meses e 6 meses. Assim respectivamente as médias foram para 1 mês 3,37\%, 7,85\% para 3 meses e 10,20\% para 6 meses. A relação para os efeitos colaterais foram que 31\% correspondendo a 9 paciente não apresentaram efeitos adversos, e $34,48 \%$ correspondendo a 10 pacientes tiveram um efeito e 7 pacientes tiveram 2 efeitos e 3 tiveram três efeitos. Não foi encontrado nenhuma relação entre a dose e o processo de continuidade do uso da liraglutida, mas afirma-se que $48 \%$ dos participantes teve aceitabilidade e satisfação completando o estudo. Onde pode ser defendido que a liraglutida é eficaz e aceito e tolerado por aproximadamente $50 \%$ dos participantes. $\mathrm{O}$ autor destaca a necessidade de uma amostra por mais dias e que seja controlado e prospectivo.

Outra característica que deve ser observada sobre a liraglutida é o seu custo, como destacado por Papamargaritis et al. (2020), que buscaram investigar a eficácia e o custo da liraglutida 3,0mg usado para tratamento a obesidade. o que foi observado é que nos países como o Reino Unido e Irlanda, há um especialista que cuida das necessidades que se insere a obesidade, assim este visa o controle de peso, que fornecem múltiplos componente e intervenções ao estilo de vida e apoio a perda de peso, incluindo o uso de medicação. Por meio de critérios individuais diversos estudos garantem a eficácia da liraglutida, mas não em um serviço de controle de peso, o qual verifica se a liraglutida é clinicamente eficaz e de baixo custo para casos graves e complexos de obesidade assim podendo melhor os serviços de combate a obesidade.

Na pesquisa de Peradze et al. (2019), que investigou o tratamento de curto prazo com alta dose liraglutida melhora o perfil de lipídios e lipoproteínase altera os mediadores hormonais de lipídios metabolismo em pacientes obesos sem manifestação diabetes mellitus tipo 2, além do alto risco de doenças cardiovasculares. Os participantes da pesquisa foram 20, obesos, durante 5 semanas. Foi verificado que os marcadores cardiovasculares mais importantes, como o colesterol total o livre e outros foram reduzidos e ajudaram na perda de peso. Outra observação é o número de tirosina reduziu e o nível de ácido biênico aumentou. foi verificado que o tratamento com alta dose de liraglutida por curto período de tempo proporcionou alterações lipídico-lipoproteico e hormonal que são sugestivas a menor riscos de doenças cardiovasculares.

Le Roux et al. (2018), investigou a eficácia e segurança da liraglutida 3,0mg entre dois grupos um com diabetes e outro sem diabetes do tipo 2 , e foi observado pontos de eficácia quanto a perda de peso, e conclui que há perda significativa no grupo que usou a liraglutida, independente se possuía ou não DM2. Com perda de até $15 \%$ para o grupo com a medicação. As melhorias significativas foram a melhora da glicemia em jejum e PA. Relatando também sobre a melhora da pontuação do IWQOL-Lite, principalmente representando a função física. É importante destacar que, na análise não foi indicado quaisquer diferenças nos efeitos do tratamento ou perfil de segurança para participantes com IMC de $27 \mathrm{a}<35 \mathrm{~kg} / \mathrm{m}^{2}$, ou seja, podendo ser recomendado a mesma dosagem para indivíduos com IMC $\geqslant 35$.

A melhoria na perda de peso, redução de massa gorda, e outros benefícios é mostrado por Kolotkin et al. (2016), que através do IWQOL-Lite e do SF-36 mensura as mudanças nos grupos, onde o destaque é para o grupo que consume a liraglutida. A escala em destaque foi a referente a dor corporal, evidencias mostram diminuição muito significativa. Concomitantemente, Wilding et al. (2016), afirma da mesma forma sobre a diferença dos grupos quanto ao estimulo ao emagrecimento, onde o grupo liraglutida é prevalente ao grupo controle em vários aspectos, como a perda de massa gorda e não de massa magra, aspecto da autoestima, e psicossocial, outros os efeitos positivos como a redução da glicose, consequentemente o controle glicêmico.

Tronieri et al. (2020), avaliaram a perda de peso por meio da adição de fentermina ao liraglutida em pacientes que já haviam perdido peso anteriormente somente com a liraglutida. Os participantes da pesquisa foram 45 adultos com obesidade e perdem média de 12,6 kg de peso inicial durante um estudo de 1 ano com liraglutida e tratamento comportamental intensivo, sendo acrescido a quantidade de $15 \mathrm{mg}$ de fentermina ou liraglutida mais placebo mais as sessões de aconselhamento durante 12 semanas. Foi relatado pelos pacientes que usaram a fentermina mais liraglutida grandes reduções na fome e na preocupação com os alimentos. A combinação foi bem tolerada, mais não induziu mais a perda de peso que antes da liraglutida.

Para Tronieri et al. (2019), muitas pesquisas são realizadas a respeito da capacidade da liraglutida de auxiliar na perda de peso corporal, mas não investigam quanto as mudanças em relação ao apetite dos participantes. Assim em 113 adultos foram avaliadas as mudanças de apetite, fatores como a fome, saciedade 
após as refeições, gostos, preocupação com a comida. O ensaio durou 52 semanas, com a dose de liraglutida de 3,0mg por dia. Na semana 52 foi observado a perda de aproximadamente $11,8 \%$ do peso inicial, que foi muito superior a somente a terapia comportamental que foi de $6,2 \%$. Em meios as semanas não houve mudanças significativas ao fim as 52 semanas, até a 24 semanas houve melhorias quanto a fome, saciedade e preocupação alimentar. O que foi certificado é sobre a efetiva perda de peso corporal dos participantes submetidos a terapia comportamental associada a liraglutida.

Na pesquisa de Chou \& Chuang (2020), a obesidade e a síndrome metabólica são conhecidas por estarem associadas por múltiplas doenças de caráter crônicas. Nas regiões asiáticas é comum o uso de increatina, em que altas doses de liraglutida não são muito utilizadas, gerando poucas avaliações a respeito do fármaco. Os autores, selecionaram 46 pacientes para realizar o estudo no intuito de avaliar a eficácia da liraglutida em baixas doses no controle de peso de pacientes, com doses de 0,6 ou 1,2 mg por dia para dedução de peso pelo período de 12 semanas. E depois deste período foram comparadas as mudanças do peso corporal, cinturas e fatores metabólicos e a redução do peso foi o fator mais evidente com a redução e $5 \%$ em 12 semanas. Destacando que as doses mais altas de liraglutida foram mais eficazes em torno de $44,4 \%$ dos pacientes, enquanto o grupo que recebeu $0,6 \mathrm{mg}$ foi em torno de $32,1 \%$ dos pacientes. Um fator determinante foi a idade, em que os mais jovens tiveram melhor chance de atingir o peso. Os autores afirmam que mesmo as baixas doses de liraglutida são eficazes na redução do peso corporal.

Wharton et al. (2020), com o objetivo de investigar se a dose de liraglutida 3,0mg é associada a perda de peso em um "mundo real", relacionando a persistência em todas as classes de obesidade em um ambiente clinico. Foi acrescentado como método uma dieta e exercícios físicos. Os pacientes foram agrupados por IMC em que a classe I (IMC 30-34,9); classe II (IMC 35-39,9); e classe III (IMC \$40). A avaliação foi realizada de acordo com a mudança de peso, perda de peso categórica, tempo para a dose de manutenção com analise de tempo e persistência para cada turma e para diferenças entre as turmas. Os desvios de classes I, II, e III respectivamente foram $-7,0 \%,-6,6 \%$ e $6,1 \%$ em seis meses. Tendo como tempo médio para a manutenção foi de 64 dias para classe I, 76 dias para a classe II, e de 71 dias para a classe III. A persistência também foi semelhante, afirmando que a liraglutida na pratica clinica é significativamente eficaz na perda de peso independente da classe de obesidade.

Park et al. (2021) a Coreia do Sul foi um dos primeiros países a provar a liraglutida como tratamento para a obesidade, e mesmo assim a droga não foi utilizada para muitas pesquisas asiáticas. $\mathrm{O}$ autor estudou respectivamente pacientes obesos com IMC maior que $27 \mathrm{~kg} / \mathrm{m} 2 \mathrm{em}$ uma clínica. As covariáveis forma o peso, IMC, hemoglobina glicada. E os dados foram coletados antes do uso da liraglutida. O prazo entre as avaliações foram de 30, 60, 90 e 180 dias. O total de pacientes na pesquisa foram 169 participantes e $42 \%$ foram do sexo masculino. O peso médio foi de $85,2 \mathrm{~kg}$, IMC médio $30,8 \mathrm{~kg} / \mathrm{m} 2$. foram observados a perda de peso nos prazos de 30 dias: 1,8kg, 60 dias: $2,3 \mathrm{~kg}, 90$ dias: 2,6kg e 180 dias: 3,5kg. Mediante a análise de composição corporal a perda de gordura foi muito maior que a de tecido muscular. Além disso, a perda de peso e não foi relacionada a idade e sexo, tabagismo, consumo de álcool e de café. Em suma, o uso da liraglutida levou a perda significativa de peso em pacientes sul-coreanos, a droga mostrou-se maior eficácia em períodos de maior usabilidade.

Os autores Wharton et al. (2019), Kolotkin et al. (2016) e Le Roux et al. (2018), afirmam a necessidade a acompanhamento rigoroso por profissionais que conheçam os efeitos da liraglutida, pois para se ter o efeito desejado deve-se dosar à cada paciente de forma individual e de acordo com as necessidades fisiológicas de cada indivíduo. Visto que altas doses pode influenciar em efeitos prejudiciais ao bem-estar, à saúde como um todo. Ou destaque se dá pela necessidade de intervenções diferentes da medicamentosa, como a pratica de exercício físico e educação alimentar, bem como em alguns casos usos de outros medicamentos, devido as condições de saúde apresentadas, como hipertensão e distúrbios renais e hepáticos.

\section{Considerações finais}

Mediante os achados, observou-se que o uso da liraglutida é eficaz para auxiliar no processo de emagrecimento, mesmo nos participantes com condições genéticas de grande influência a obesidade, como para adolescentes e crianças, a precaução e cuidados é quanto a dosagem que na maioria a variação foi de 0,6 a 3,0mg de liraglutida, com destaque que geralmente as doses mais altas foram mais efetivas, nenhuma pesquisa relatou utilizar mais que 3,0mg do fármaco.

Outros achados foram quanto aos efeitos secundários, como melhora da PA e o controle do nível de glicêmico, além de melhorar a autoestima e a funcionalidade de indivíduos associando a melhora 
comportamental com os alimentos. E melhoras quanto a condições geradas pela obesidade com a apneia do sono, colesterol, triglicerídeos e problemas cardiovasculares em geral.

Também foi evidenciado efeitos adversos principalmente de origem gastrointestinais, independente do público, pois se manifestaram em crianças, adolescente e adultos, independentes da dosagem porque mesmo em doses mais baixas alguns participantes relataram incômodos gerados pelo medicamento, no entanto foi considera de alta tolerabilidade e segurança.

Os autores foram unânimes quanto a eficácia da liraglutida para o processo de emagrecimento, mesmo em populações que não tinham costumes de indicação da droga como os asiáticos, especialmente os sul-coreanos. O fármaco foi eficaz também em estudos de curta durabilidade e doses mais altas, como que estudos mais prolongados com doses mais baixas, não tendo interferências significativas quanto a classe de obesidade. Outro fator a destacar é a associação com três intervenções mais utilizadas que são a terapia comportamental intensiva, dieta de déficit calórico e associação com um programa de exercício físico.

Assim, diversos autores destacam a necessidade de mais amostragens para que se possa mensurar esses efeitos, em públicos diferentes com dosagens e espaço-tempo também diferentes. Notou-se que o uso da liraglutida é eficiente desde que seja ministrado de forma consciente e em doses equilibradas baseadas no histórico e conhecimento do perfil do paciente.

\section{Referências}

Agência Nacional de Vigilância Sanitária - ANVISA. (2017). Liraglutida é aprovada como tratamento auxiliar para o controle do peso em adultos - Português (Brasil). https://www.gov.br/anvisa/pt-br/assuntos/noticiasanvisa/2016/liraglutida-e-aprovada-como-tratamento-auxiliar-para-o-controle-do-peso-em-adultos.

Arruda, A.C., Izolan, N.F., Godoy, M.K., Kupske J.W., Horn, R.C., \& Herk, T.G. 2019. Uso de liraglutida associada ao exercício físico no combate a obesidade. Bioeconomia: diversidade e riqueza para o desenvolvimento sustentável. Salão do conhecimento. Disponível em: https://publicacoeseventos.unijui.edu.br/index.php/salaoconhecimento/article/view/11683

Barreto, F. M. (2013). Perda de Peso com Incretinomiméticos Experiência Profissionalizante na vertente de Farmácia Comunitária e Investigação. Dissertação (Mestrado) - Curso de Ciências Farmacêuticas, Universidade da Beira Interior, Covilhã, 84p.

Blackman, A., Foster, G. D., Zammit, G., Rosenberg, R., Aronne, L., Wadden, T., Claudius, B., Jensen, C. B., \& Mignot, E. (2016). Effect of liraglutide $3.0 \mathrm{mg}$ in individuals with obesity and moderate or severe obstructive sleep apnea: The scale sleep apnea randomized clinical trial. International Journal of Obesity, 40(8), 1310-1319.

Brasil. 2012. Conselho Nacional de Saúde. Resolução nº466, de 12 de dezembro de 2012. Brasília, DF .Disponível em: http://www.conselho.saude.gov.br/web_comissoes/conep/index.html

Brito, C. F., Lima, E. R., Oliveira, J. A., Pitol, L. C., Campos, N. T., \& Rabelo, F. 2013. Mecanismo de ação das incretinas e o potencial terapêutico de moléculas relacionadas no tratamento do diabetes Mellitus tipo 2. NOVA: Revista Científica, 2(2), 1-20.

Chao, A. M., Wadden, T. A., Walsh, O. A., Gruber, K. A., Alamuddin, N., Berkowitz, R. I., \& Tronieri, J. S. (2019). Effects of Liraglutide and Behavioral Weight Loss on Food Cravings, Eating Behaviors, and Eating Disorder Psychopathology. Obesity, 27(12), 2005-2010.

Chou, C. A., \& Chuang, S. F. (2020). Evaluation of the efficacy of low-dose liraglutide in weight control among Taiwanese non-diabetes patients. Journal of Diabetes Investigation, 11(6), 1524-1531.

Cuomo, A., Bolognesi, S., Goracci, A., Ciuoli, C., Crescenzi, B. B., Maina, G., Rosso, G., Facchi, E., Maccora, C., Giordano, N., Verdino, V., \& Fagiolini, A. (2019). Feasibility, adherence and efficacy of liraglutide treatment in a sample of individuals with mood disorders and obesity. Frontiers in Psychiatry, 10(JAN), 784.

Danne, T., Biester, T., Kapitzke, K., Jacobsen, S. H., Jacobsen, L. V., Petri, K. C. C., Hale, P. M., \& Kordonouri, O. (2017). Liraglutide in an Adolescent Population with Obesity: A Randomized, Double-Blind, PlaceboControlled 5-Week Trial to Assess Safety, Tolerability, and Pharmacokinetics of Liraglutide in Adolescents Aged 12-17 Years. Journal of Pediatrics, 181, 146-153.e3.

Fujioka, K., O’Neil, P. M., Davies, M., Greenway, F., C.W. Lau, D., Claudius, B., Skjøth, T. V., Bjørn Jensen, C., \& P.H. Wilding, J. (2016). Early Weight Loss with Liraglutide $3.0 \mathrm{mg}$ Predicts 1-Year Weight Loss and is Associated with Improvements in Clinical Markers. Obesity, 24(11), 2278-2288. 
Iepsen, E. W., Zhang, J., Thomsen, H. S., Hansen, E. L., Hollensted, M., Madsbad, S., Hansen, T., Holst, J. J., Holm, J. C., \& Torekov, S. S. (2018). Patients with Obesity Caused by Melanocortin-4 Receptor Mutations Can Be Treated with a Glucagon-like Peptide-1 Receptor Agonist. Cell Metabolism, 28(1), 23-32.e3.

Júnior, A. V. V., Gomes, C. F., Allgayer, N., \& Lara, G. M. (2012). A grelina e sua contribuição para obesidade e Diabetes Mellitus tipo 2. Revista Conhecimento Online, 2, 1-8.

Kochar, I. S., \& Sethi, A. (2019). Efficacy and safety of liraglutide in Indian adolescents with obesity. Obesity Science and Practice, 5(3), 251-257.

Kolotkin, R. L., Fujioka, K., Wolden, M. L., Brett, J. H., \& Bjorner, J. B. (2016). Improvements in health-related quality of life with liraglutide $3.0 \mathrm{mg}$ compared with placebo in weight management. Clinical Obesity, 6(4), 233-242.

Kolotkin, R. L., Gabriel Smolarz, B., Meincke, H. H., \& Fujioka, K. (2018). Improvements in health-related quality of life over 3 years with liraglutide $3.0 \mathrm{mg}$ compared with placebo in participants with overweight or obesity. Clinical Obesity, 8(1), 1-10.

Le Roux, C., Aroda, V., Hemmingsson, J., Cancino, A. P., Christensen, R., \& Pi-Sunyer, X. (2018). Comparison of Efficacy and Safety of Liraglutide $3.0 \mathrm{mg}$ in Individuals with BMI above and below $35 \mathrm{~kg} / \mathrm{m} 2$ : A Posthoc Analysis. Obesity Facts, 10(6), 531-544.

Mastrandrea, L. D., Witten, L., Carlsson Petri, K. C., Hale, P. M., Hedman, H. K., \& Riesenberg, R. A. (2019). Liraglutide effects in a paediatric (7-11 y) population with obesity: A randomized, double-blind, placebocontrolled, short-term trial to assess safety, tolerability, pharmacokinetics, and pharmacodynamics. Pediatric Obesity, 14(5).

Morante, Y. M., \& Galende, S. B. (2016). Mecanismo de ação da liraglutida em pacientes acometidos por diabetes Mellitus tipo 2. Uningá Review Journal, 25(1), 74-77.

Neres, M. S., Netto, P. A., \& Gusmão, R. V. (2019). A liraglutida no tratamento da obesidade. Faculdade Unida de Campinas.

Overgaard, R. V., Petri, K. C., Jacobsen, L. V., \& Jensen, C. B. (2016). Liraglutide $3.0 \mathrm{mg}$ for Weight Management: A Population Pharmacokinetic Analysis. Clinical Pharmacokinetics, 55(11), 1413-1422.

Papamargaritis, D., Al-Najim, W., Lim, J., Crane, J., Lean, M., Le Roux, C., McGowan, B., O’Shea, D., Webb, D., Wilding, J., \& Davies, M. J. (2020). Effectiveness and cost of integrating a pragmatic pathway for prescribing liraglutide $3.0 \mathrm{mg}$ in obesity services (STRIVE study): Study protocol of an open-label, realworld, randomised, controlled trial. BMJ Open, 10(2), e034137.

Papathanasiou, T., Strathe, A., Agersø, H., Lund, T. M., \& Overgaard, R. V. (2020). Impact of dose-escalation schemes and drug discontinuation on weight loss outcomes with liraglutide $3.0 \mathrm{mg}$ : A model-based approach. Diabetes, Obesity and Metabolism, 22(6), 969-977.

Park, J. S., Kwon, J., Choi, H. J., \& Lee, C. (2021). Clinical effectiveness of liraglutide on weight loss in South Koreans: First real-world retrospective data on Saxenda in Asia. Medicine, 100(2), e23780.

Peradze, N., Farr, O. M., Perakakis, N., Lázaro, I., Sala-Vila, A., \& Mantzoros, C. S. (2019). Short-term treatment with high dose liraglutide improves lipid and lipoprotein profile and changes hormonal mediators of lipid metabolism in obese patients with no overt type 2 diabetes mellitus: A randomized, placebo-controlled, cross-over, double-blind clinical trial. Cardiovascular Diabetology, 18(1), 1-12.

Pereira, J.M.M.S. 2010. Incretinas: actualização do conceito, novas moléculas e indicações. Dissertação de Mestrado em Medicina. Faculdade de Medicina da Universidade de Coimbra. Disponível em: https://estudogeral.uc.pt/handle/10316/45602

Ramos, E. V., \& Arjona, M. D. C. C. 2013. Características clínicas y frecuencia de complicaciones crónicas en personas con diabetes mellitus tipo 2 de diagnóstico reciente. Revista Cubana de Medicina General Integral, 29(2).

Silva, L. F. O., da Silva, F. V. M., \& Oyama, S. M. R. 2013. Prevalência do uso de medicamentos para emagrecer entre universitárias. Revista Recien-Revista Científica de Enfermagem, 3(7), 19-26.

Souza, N. B. (2012). Efeito das incretinas no tratamento do diabetes Mellitus tipo 2. Universidade do Extremo Sul Catarinense. Monografia (Graduação) - Curso de Farmácia, Universidade do Extremo Sul Catarinense, Criciúma, 67p.

Tronieri, J. S., Wadden, T. A., Walsh, O. A., Berkowitz, R. I., Alamuddin, N., Gruber, K., Leonard, S., \& Chao, A. M. (2019). Effects of liraglutide plus phentermine in adults with obesity following 1 year of treatment by liraglutide alone: A randomized placebo-controlled pilot trial. Metabolism: Clinical and Experimental, 


$$
\text { 96, 83-91. }
$$

Tronieri, J. S., Wadden, T. A., Walsh, O., Berkowitz, R. I., Alamuddin, N., Gruber, K., Leonard, S., Bakizada, Z. M., \& Chao, A. M. (2020). Effects of liraglutide on appetite, food preoccupation, and food liking: results of a randomized controlled trial. International Journal of Obesity, 44(2), 353-361.

Wharton, S., Haase, C. L., Kamran, E., Liu, A., Mancini, J., Neish, D., Pakseresht, A., Power, G. S., \& Christensen, R. A. G. (2020). Weight loss and persistence with liraglutide $3.0 \mathrm{mg}$ by obesity class in the real-world effectiveness study in Canada. Obesity Science and Practice, 6(4), 439-444.

Wharton, S., Liu, A., Pakseresht, A., Nørtoft, E., Haase, C. L., Mancini, J., Power, G. S., Vanderlelie, S., \& Christensen, R. A. G. (2019). Real-World Clinical Effectiveness of Liraglutide $3.0 \mathrm{mg}$ for Weight Management in Canada. Obesity, 27(6), 917-924.

World Health Organization - WHO. (2021). Obesity and overweight. https://www.who.int/news-room/factsheets/detail/obesity-and-overweight

Wilding, J. P. H., Overgaard, R. V., Jacobsen, L. V., Jensen, C. B., \& Le Roux, C. W. (2016). Exposure-response analyses of liraglutide 3.0mg for weight management. Diabetes, Obesity and Metabolism, 18(5), 491-499.

\section{Minicurrículo}

Thales Henrique Martins Araújo. Acadêmico do curso de Farmácia da Faculdade de Palmas - FAPAL.

Ciro José de Sousa Carvalho. Graduado em Medicina Veterinária pela UFPI, Mestre em Ciência Animal com ênfase em toxicologia pela UFPI com participação em Programa de Cooperação Acadêmica (PROCAD) pela UFMG e Doutorado em Ciência Animal com ênfase em Diagnóstico precoce de metástases em câncer de mama pela UFPI.

Como citar: Araújo, T.H.M, \& Carvalho, C.J.S. 2021. Expectativa da Ação da Liraglutida no Processo de Emagrecimento. Pubsaúde, 8, a169. DOI: https://dx.doi.org/10.31533/pubsaude8.a169

Recebido: 25 abr. 2021.

Revisado e aceito: 4 mai. 2021.

Conflito de interesse: os autores declaram, em relação aos produtos e companhias descritos nesse artigo, não ter interesses associativos, comerciais, de propriedade ou financeiros que representem conflito de interesse.

Licenciamento: Este artigo é publicado na modalidade Acesso Aberto sob a licença Creative Commons Atribuição 4.0 (CC-BY 4.0). 